Published in final edited form as:

Breast Cancer Manag. 2014 May ; 3(3): 227-228. doi:10.2217/bmt.14.13.

\title{
Our panel of experts highlight the most important research articles across the spectrum of topics relevant to the field of breast cancer management
}

\author{
Lawrence J Solin, \\ Albert Einstein Healthcare Network, PA, USA \\ Pauline T Truong, \\ University of British Columbia, BC, Canada \\ Jose Russo, \\ Fox Chase Cancer Center, PA, USA \\ Hitoshi Tsuda, \\ National Defense Medical College, Japan \\ Cesar A Santa-Maria, and \\ The Johns Hopkins Kimmel Cancer Center, MD, USA \\ Antonio C Wolf \\ The Johns Hopkins Kimmel Cancer Center, MD, USA
}

\section{Wong JS, Chen YH, Gadd MA et al. Eight-year update of a prospective study of wide excision alone for small low- or intermediate-grade ductal carcinoma in situ (DCIS). Breast Cancer Res. Treat. 143, 343-350 (2014)}

\begin{abstract}
The optimal treatment of patients with ductal carcinoma in situ (DCIS) of the breast remains controversial, with large variations in clinical management. Wong et al. report the updated results of their trial of wide local excision alone for 158 patients with DCIS. Wide negative margins of resection were required. Radiation treatment and tamoxifen were not given. The 10-year rate of local recurrence was $15.6 \%$. Of the local recurrences, $32 \%$ were invasive. The 10-year rate of local recurrence was similar to the findings from the ECOG E5194 study.

The results reported by Wong et al. demonstrate that even for patients with clinical and pathologic features suggesting favorable disease, there is a substantial and continuing rate of local recurrence, including invasive local recurrence. Because of the need to improve risk
\end{abstract}

\footnotetext{
For reprint orders, please contact: reprints@ futuremedicine.com

Financial \& competing interests disclosure: The authors have no relevant affiliations or financial involvement with any organization or entity with a financial interest in or financial conflict with the subject matter or materials discussed in the manuscript. This includes employment, consultancies, honoraria, stock ownership or options, expert testimony, grants or patents received or pending, or royalties.

No writing assistance was utilized in the production of this manuscript.
} 
stratification beyond conventional clinical and pathologic parameters, molecular tools, such as the Oncotype DX ${ }^{\circledR}$ (Genomic Health, Inc., CA, USA) DCIS score, will become increasingly important in clinical practice.

- Written by Lawrence J Solin

\section{Veronesi U, Orecchia R, Maisonneuve P et al. Intraoperative radiotherapy versus external radiotherapy for early breast cancer (ELIOT): a randomized controlled equivalence trial. Lancet Oncol. 14(13), 1269 -1277(2013)}

In this trial from the European Institute of Oncology, 1305 women aged 48-75 years with tumors $<2.5 \mathrm{~cm}$ were randomly assigned to whole-breast radiation therapy delivering 50 Gy/25 fractions plus boost $10 \mathrm{~Gy} /$ five fractions versus intraoperative radiation therapy (IORT) delivering a single fraction of $21 \mathrm{~Gy}$ to the tumor bed after quadrantectomy. An equivalence margin was prespecified as 5-year in-breast tumor recurrence not exceeding $7.5 \%$ in the IORT group. At a median follow-up of 5.8 years, in-breast tumor recurrence was significantly higher in the IORT compared with the whole-breast radiation therapy group (4.4 vs $0.4 \%$; hazard ratio: 9.3 ; $95 \%$ CI: $3.3-26.3$ ). Among 876 subjects with available data, IORT was associated with fewer skin toxicity $(\mathrm{p}=0.0002)$, but higher rates of fat necrosis ( $\mathrm{p}$ $=0.04)$. The findings that IORT was associated with suboptimal local control teach important lessons about exercising caution in not replacing standard therapy with newer methods until results from rigorous prospective evaluation are available.

- Written by Pauline T Truong

\section{Kazi AA, Gilani RA, Schech AJ et al. Nonhypoxic regulation and role of hypoxia-inducible factor 1 in aromatase inhibitor resistant breast cancer. Breast Cancer Res. 16(1), R15 (2014)}

In this article, a new twist on the resistant of breast cancer patients to aromatase inhibitors (AIs; for example, letrozole) is presented. It has been suggested in the published literature that acquired resistance to AIs involves a switch from dependence on estrogen receptor signaling to dependence on growth factor-mediated pathways, such as HER2. However, the role of HER2, and the identity of other relevant factors that may be used as biomarkers or therapeutic targets remain unknown. In the present work, the investigators explored the potential role of transcription factor HIF-1 in acquired AI resistance, and its regulation by HER2. In conclusion, one of the mechanisms of AI resistance may be through the regulation of nonhypoxic HIF-1 target genes, such as BCRP, implicated in chemoresistance. Thus, $H I F-1$ should be explored further for its potential as a biomarker of and therapeutic target.

- Written by Jose Russo 


\section{Visscher DW, Nassar A, Degnim AC et al. Sclerosing adenosis and risk of breast cancer. Breast Cancer Res. Treat. 144, 205-212 (2014)}

Sclerosing adenosis (SA) is a common proliferative lesion seen in fibrocystic changes. In Jensen's 'classic' work (1989) [1], the relative risk of SA for breast cancer was 2.1 in the study of 349 women. SAs are detected in both premenopausal and postmenopausal women and in association with various histological features. In this large-scale study of 13,434 biopsies, the risk ratio (2.10) of SA was similar to that of Jensen. SA was associated with increased breast cancer risk in all age groups ( $<45,45-55$ and $>55$ years) and in both groups with no $(0 \%)$ and partial (1-74\%) lobular involution, but did not influence on the risk in specimens with proliferative lesions with or without atypia.

- Written by Hitoshi Tsuda

\section{Aebi S, Gelber S, Anderson SJ et al. Chemotherapy for isolated locoregional recurrence of breast cancer (CALOR): a randomised trial. Lancet Oncol. 15(2), 156-63 (2014)}

Isolated locoregional recurrences from breast cancer are associated with high recurrence rates and breast cancer-related death even after local therapy. The role of additional chemotherapy after local treatment of isolated locoregional recurrences has been controversial; however, investigators in the CALOR trial explore this question. Patients with isolated locoregional recurrences were randomized to chemotherapy (providers choice) versus a control arm (no chemotherapy), and a total of 162 patients were accrued from 2003 to 2010. The chemotherapy arm was found to have improved disease-free survival compared with controls (69 vs 57\%, hazard ratio: 0.59 [95\% CI: 0.35-0.99]; $\mathrm{p}=0.046$ ); however, this was only significant in patients estrogen receptor-negative tumors $(\mathrm{p}=0.046)$. While the study is limited by a small sample size, it provides evidence that patients should consider chemotherapy after local therapy of isolated locoregional recurrences, particularly if they have estrogen receptor-negative tumors.

- Written by Cesar A Santa-Maria \& Antonio C Wolf

\section{Travier N, Fonseca-Nunes A, Javierre C et al. Effect of a diet and physical activity intervention on body weight and nutritional patterns in overweight and obese breast cancer survivors. Med. Oncol. 31(1), 783 (2014)}

Most women diagnosed with breast cancer in the USA are overweight or obese, and are likely to gain weight with treatment. Both factors result in increased risk of breast cancer recurrence and death; however, there is currently no standard-of-care weight management program for breast cancer survivors. This Phase II single-arm prospective trial enrolled 42 women with early-stage breast cancer and a BMI of $\geq 25 \mathrm{~kg} / \mathrm{m}^{2}$ to undergo an in-person diet and exercise weight loss intervention for 12 weeks. The 37 women who completed the study lost an average of $5.6 \pm 2.0 \mathrm{~kg}$, and had improvements in dietary behavior, quality of life and cardiorespiratory fitness measures. These results confirm that weight loss is feasible in 
breast cancer survivors; however, larger randomized trials utilizing scalable interventions investigating modulation in biomarkers of energy dynamics and cancer risk are urgently needed in order to develop weight loss as a therapy for primary and secondary breast cancer prevention.

\author{
- Written by Cesar A Santa-Maria \& Antonio C Wolf
}

\title{
References
}

1. Jensen RA, Page DL, Dupont WD, Rogers LW. Invasive breast cancer risk in women with sclerosing adenosis. Cancer. 1989; 64(10):1977-1983. [PubMed: 2804888] 